\title{
О СТРУКТУРЕ СПЛАВНЫХ ГЕТЕРОПЕРЕХОДОВ В ЭВТЕКТИЧЕСКИХ СИСТЕМАХ
}

\section{Введение}

В [1] рассмотрена зависимость строения многослойных структур, образующихся при изготовлении сплавных гетеропереходов в системах с вырожденной эвтектикой, от ряда параметров. Показано, что наличие, направление и величина градиента температуры при сплавлении, длительность сплавления, направление отвода теплоты, величина градиента температуры при кристаллизации, а также скорость охлаждения оказывают определяющее влияние на строение слоистой структуры. В настоящей работе методом, описанным в [1], рассмотрена зависимость строения слоистых структур от перечисленных выше параметров в случае, когда сплавляемые вещества образуют эвтектическую систему. Следует отметить, что образование эвтектических систем наблюдается в практике получения гетеропереходов (например $\mathrm{GaAs}-\mathrm{Ge}\left[{ }^{2-4}\right]$ ) наряду с образованием систем, дающих твердые растворы (например $\mathrm{GaAs}-\mathrm{Ga}_{x} \mathrm{Al}_{1-x} \mathrm{As}$, достоинства которой доказаны в $\left.\left[{ }^{5-7}\right]\right)$.

\section{Строение слоистых структур}

Как показано в $\left.{ }^{8}\right]$, в эвтектических системах структуры, которые образуются при изготовлении сплавных гетеропереходов, содержат двухфазные слои, имеющие близкий к эвтектическому состав. Закономерное срастание двухфазного слоя с первоначальным, по-видимому, весьма затруднено. Несмотря на это обстоятельство в нашей работе все границы слоев, где происходит нарастание нового слоя независимо от его характера на слой, который, вероятно, является монокристаллическим и благоприятно ориентированным, обозначены как границы, имеющие некоторую вероятность образования гетероперехода.

С целью выяснить особенности образования слоистых структур в эвтектических системах рассмотрим некоторые характерные примеры.

$$
\text { 1. } T_{a}>T_{A}
$$

1) $T_{a}=T_{b}$, общее понижение температуры, $0<\tau<\tau_{a b}$ (рис. $1 a$ ). При охлаждении сначала создается переохлаждение вещества $B$ у границы $L \mid B$, затем происходит направленная первичная кристаллизация автоэпитаксиального слоя $B^{\prime}$. При понижении температуры ниже $T_{A}$ создается переохлаждение вещества $A$ в слое от границы - $L$ до фронта диффузии вещества $B$, затем происходят ненаправленная кристаллизация слоя и далее направленная первичная кристаллизация вещества $A$ 
a
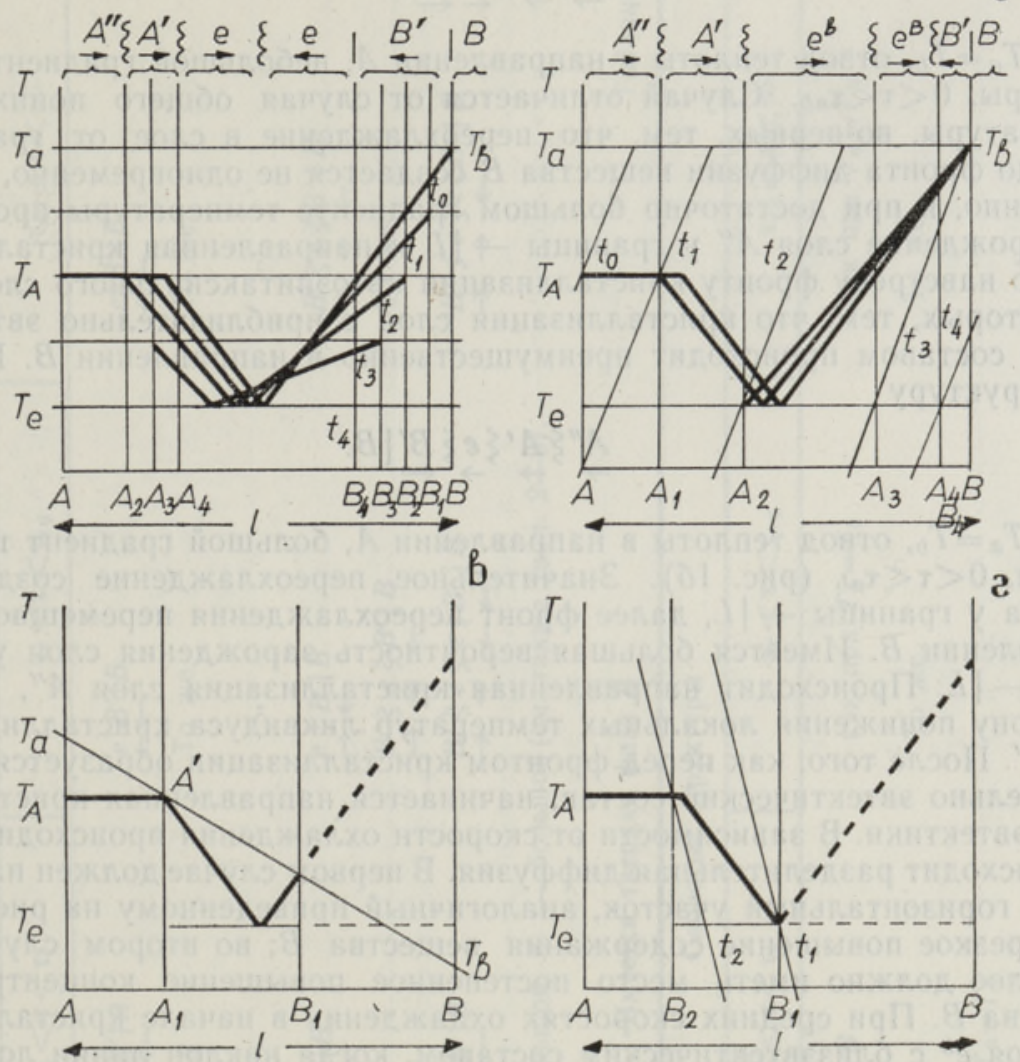

Рис. 1. Кристаллизация слоя неравновесного расплава. Эвтектика. $a-T_{a}=T_{b} ; 0<\tau<\tau_{a b} ;$ общее понижение температуры; 6 $T_{a}=T_{b} ; 0<\tau<\tau_{a b} ;$ отвод теплоты в направлении $A$; большой градиент температуры; $8-0<\tau<\tau_{a b}$; отвод теплоты в направлении $B$; небольшой граднент температуры; $2-0<\tau<\tau_{a b}$; отвод теплоты в направлении $B$; большой градиент температуры.

в сторону понижения локальных температур ликвидуса из расплава, содержащего как вещество $A$, так и вещество $B$. Как и в системах с вырожденной эвтектикой [1], первичная кристаллизация в сторону понижения локальных температур ликвидуса приводит к уменьшению наклонов линий локальных ликвидусов. Когда наклоны этих линий приближаются к нулю, происходит одновременная кристаллизация слоя, имеющего приблизительно эвтектический состав. Напомним, что при быстром охлаждении может происходить бездиффузионная кристаллизация [ $\left.{ }^{4}\right]$, которая начинается до приближения наклонов линий локальных ликвидусов к.нулю. Если обозначим слой с составом, близким к эвтектическому, через $e$, получим слоистую структуру

$$
A^{\prime \prime} \underset{A^{\prime}}{\rightarrow} e \underset{B^{\prime} \mid B}{\leftarrow}
$$

Под влиянием кристаллических слоев $A^{\prime}$ и $B^{\prime}$ кристаллизация слоя $e$ происходит, очевидно, направленно двумя фронтами, движущимися друг другу навстречу. С учетом этого явления получим структуру 


\section{$\left.A^{\prime \prime}\right\} \underset{A^{\prime}}{\rightarrow} \underset{\rightarrow}{\rightarrow}\left\{e\left|B^{\prime}\right| B\right.$}

2) $T_{a}=T_{b}$, отвод теплоты в направлении $A$, небольшой градиент температуры, $0<\tau<\tau_{a b}$. Случай отличается от случая общего понижения температуры, во-первых, тем, что переохлаждение в слое от границы - $\mid L$ до фронта диффузии вещества $B$ создается не одновременно, а направленно, и при достаточно большом градиенте температуры происходят зарождение слоя $A^{\prime \prime}$ у границы - $L$ и направленная кристаллизация его навстречу фронту кристаллизации автоэпитаксиального слоя $B^{\prime}$, и, во-вторых, тем, что кристаллизация слоя с приблизительно эвтектическим составом происходит преимущественно в направлении $B$. Получим структуру

$$
\underset{A^{\prime \prime}}{\rightarrow} \stackrel{A^{\prime}}{\rightarrow} \underset{\rightarrow}{\rightarrow} \underset{B^{\prime}}{\leftarrow} \mid B
$$

3) $T_{a}=T_{b}$, отвод теплоты в направлении $A$, большой градиент температуры, $0<\tau<\tau_{a b}$ (рис. 1б). Значительное переохлаждение создается сначала у границы - $\mid L$, далее фронт переохлаждения перемещается в направлении $B$. Имеется большая вероятность зарождения слоя у границы - $\mid L$. Происходит направленная кристаллизация слоя $A^{\prime \prime}$, затем в сторону понижения локальных температур ликвидуса кристаллизация слоя $A^{\prime}$. После того, как перед фронтом кристаллизации образуется приблизительно эвтектический состав, начинается направленная кристаллизация эвтектики. В зависимости от скорости охлаждения происходит или не происходит разделительная диффузия. В первом случае должен наблюдаться горизонтальный участок, аналогичный приведенному на рис. 4 , и затем резкое повышение содержания вещества $B$; во втором случае в этом слое должно иметь место постепенное повышение концентрации вещества $B$. При средних скоростях охлаждения в начале кристаллизации слоя $e^{b}$ с близэвтектическим составом, когда наклон линии локальных ликвидусов меньше наклона линии температур (от $t_{2}$ до $t_{3}$; рис. 1б), по-видимому, происходит все увеличивающийся захват избыточного вещества $B$. Когда наклоны этих линий совпадают, во всем жидком слое создается переохлаждение вещества $B$. Возможен рост автоэпитаксиального слоя $B^{\prime}$ на исходное вещество $B$, а также при достаточно медленном охлаждении зарождение и рост отдельных кристаллов вещества $B$ в объеме расплава. Обозначим последний слой $e^{B}$. На границе слоев $e^{b}$ и $e^{B}$ на диаграмме расстояние-состав возможен излом кривой состава. Без учета некоторого различия слоев $e^{b}$ и $e^{B}$ получим структуру

$$
\stackrel{A^{\prime \prime}}{\rightarrow} \underset{A^{\prime}}{\rightarrow} \stackrel{\xi e}{\rightarrow} \underset{\leftarrow}{\leftarrow} \mid B
$$

которая отличается от полученной ранее тем, что слой $B^{\prime}$ имеет меньшую толщину.

4) $T_{a}=T_{b}$, отвод теплоты в направлении $B$, небольшой градиент температуры, $0<\tau<\tau_{a b}$ (рис. 18). На определенной стадии кристаллизации около точки $A^{\prime}$ создается местное переохлаждение вещества $A$; имеется вероятность образования зародышей и начала кристаллизации в объеме расплава. В направлении $A$ кристаллизуется слой $A^{\prime \prime}$ и в направлении $B-$ слой $A^{\prime}$. Получим структуру

$$
\underset{\leftarrow}{A^{\prime \prime}} \underset{\leftarrow}{\leftarrow} A^{\prime} \underset{\leftarrow}{e}\left|B^{\prime}\right| B .
$$

При резком градиенте температуры (рис. 12) не происходит местного 


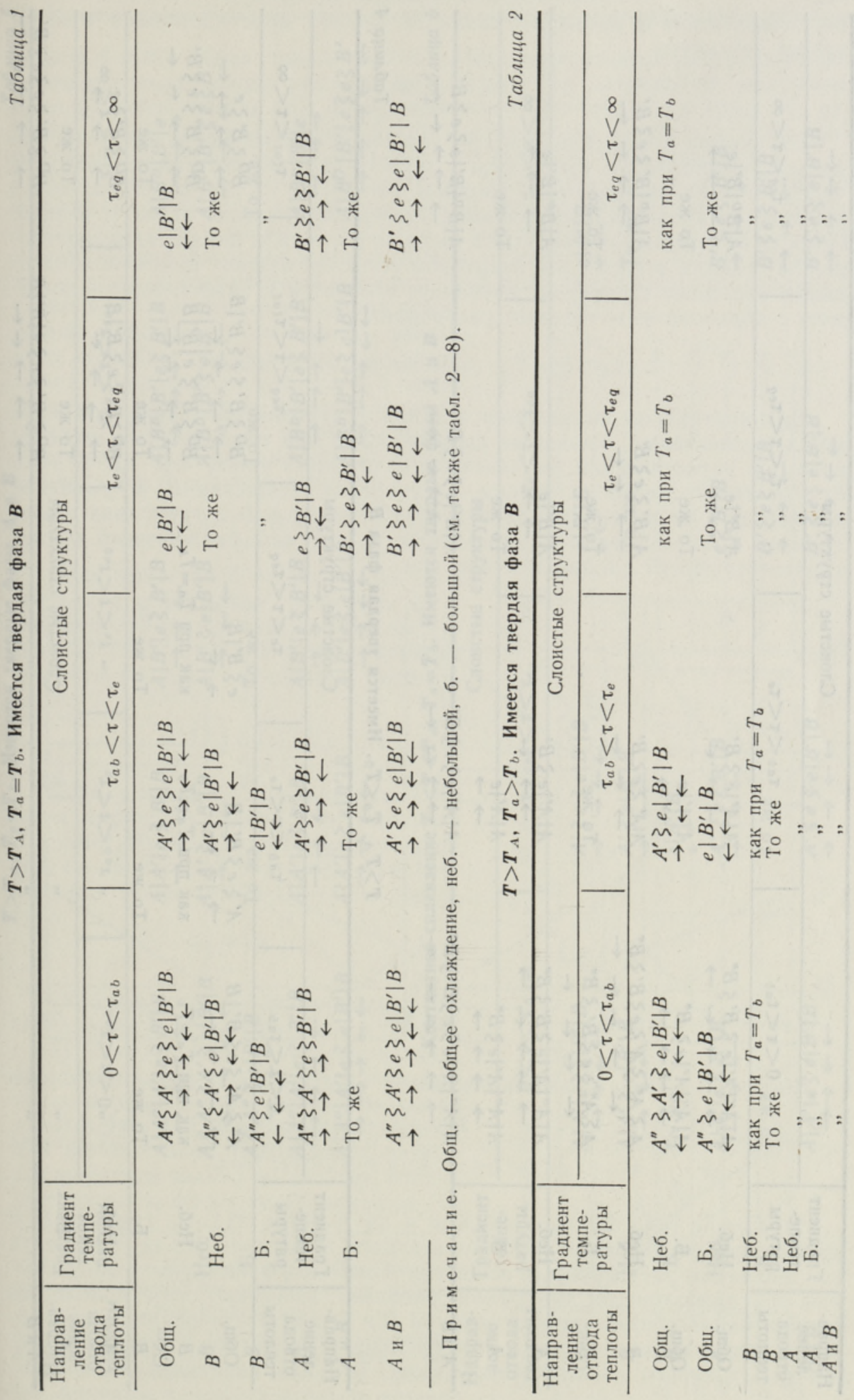




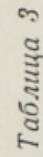

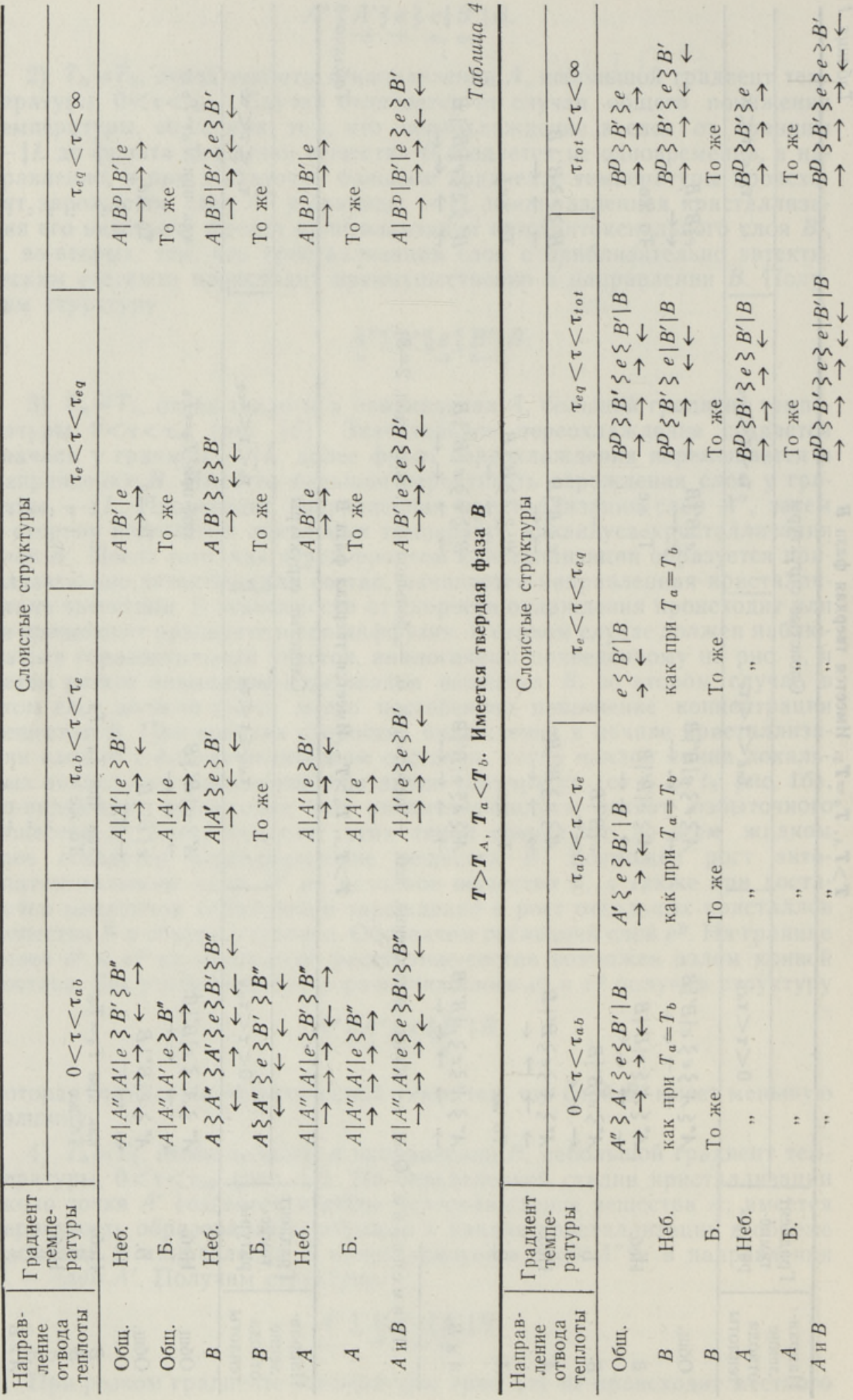




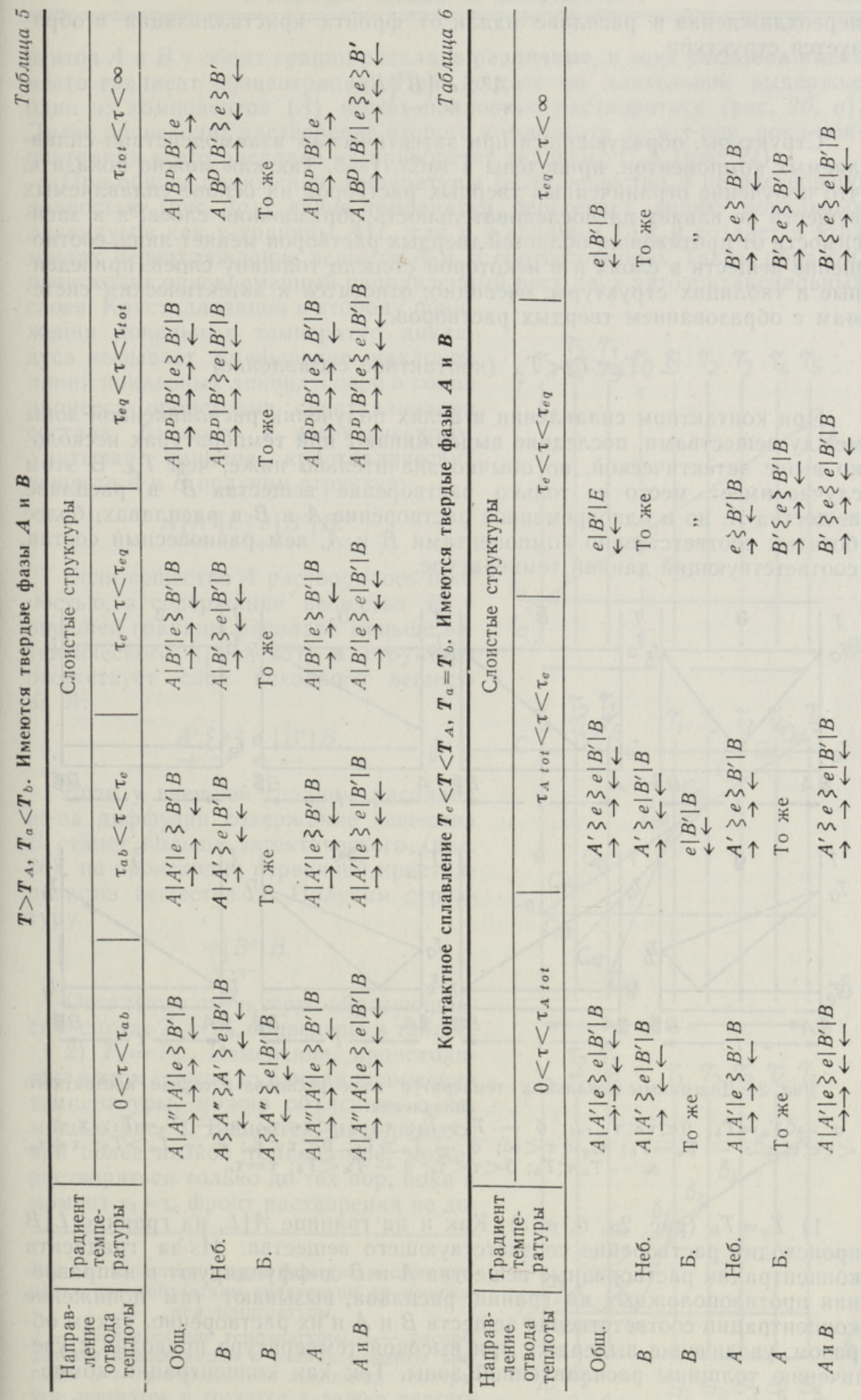


переохлаждения в расплаве вдали от фронта кристаллизации и образуется структура

$$
\underset{\leftarrow}{A^{\prime \prime}} \underset{\leftarrow}{\xi} e \underset{\leftarrow}{\leftarrow}\left|B^{\prime}\right| B
$$

Структуры, образующиеся при эвтектическом взаимодействии сплавляемых компонентов, приведены в табл. $1-5$. Так как можно показать, что получение ограниченных твердых растворов на основе сплавляемых веществ не влияет на последовательность образования слоев, а в зависимости от протяжения областей твердых растворов меняет лишь соотношение веществ в слоях и в некоторой степени толщину слоев, приведенные в таблицах структуры, очевидно, относятся к эвтектическим системам с образованием твердых растворов.

\section{2. $T_{e}<T_{a}<T_{A}$ (контактное сплавление)}

При контактном сплавлении в целях получения расплавленной зоны между веществами, последние выдерживают при температурах несколько выше эвтектической, но обычно значительно ниже, чем $T_{A}$. В этом случае имеет место не только растворение вещества $B$ в расплаве вещества $A$, но и одновременное растворение $A$ и $B$ в расплавах, более богатых соответственно компонентами $B$ и $A$, чем равновесный состав, соответствующий данной температуре.
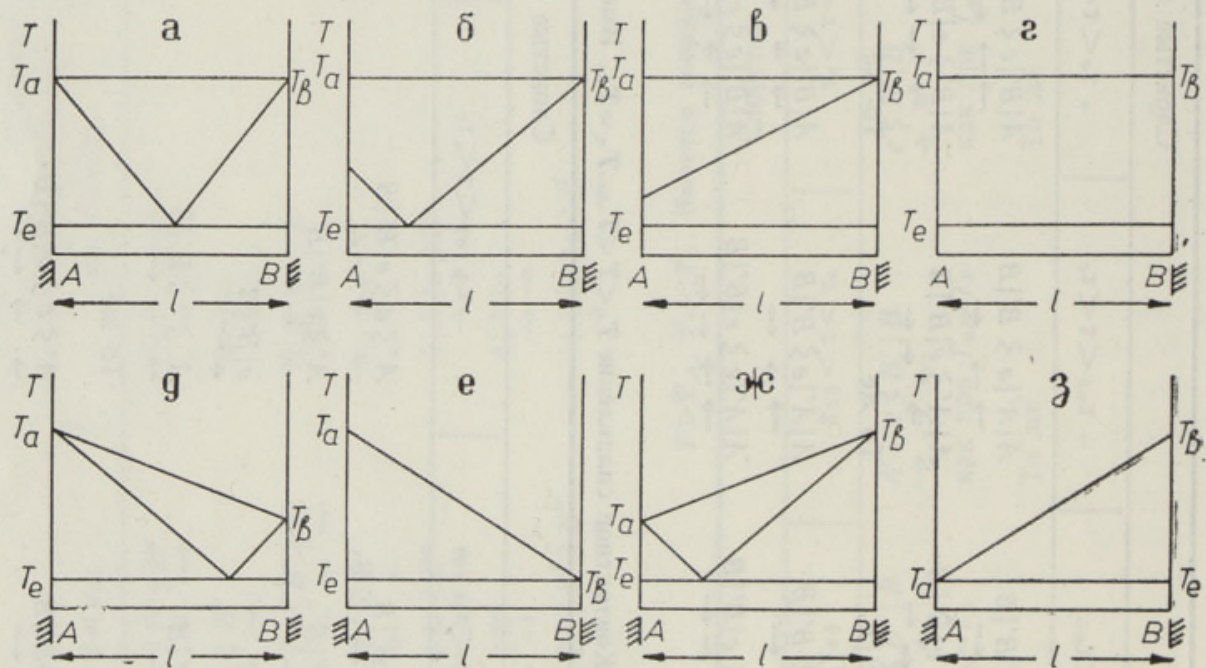

Рис. 2. Диаграммы локальных температур слоя расплава в случае контактного сплавления.

$a-T_{a}=T_{b} ; 0<\tau<\tau_{A} t_{o t} ; \sigma-T_{a}=T_{b} ; \tau_{A t o t}<\tau<\tau_{e} ; \quad$ B $-T_{a}=T_{b} ; \tau_{e}<$ $<\tau<\tau_{e q} ; 2-T_{a}=T_{b} ; \tau_{e q}<\tau<\infty ; \partial-T_{a}>T_{b} ; 0<\tau<\tau_{e} ; e-T_{a}>T_{b} ; \quad \tau=\tau_{e} ;$ $\mathscr{H}-T_{a}<T_{b} ; 0<\tau<\tau_{e} ; 3-T_{a}<T_{b} ; \tau=\tau_{e}$.

1) $T_{a}=T_{b}$ (рис. $\left.2 a, \sigma, 8,2\right)$. Как и на границе $A \mid L$, на границе $L \mid B$ происходит растворение соответствующего вещества. Из-за градиента концентрации растворенные вещества $A$ и $B$ диффундируют в направлении противоположных им границ расплава, вызывают там понижение концентрации соответственно веществ $B$ и $A$ и их растворение. Таким образом, увеличение выдержки при высокой температуре приводит к увеличению толщины расплавленной зоны. Так как концентрации компо- 
нентов $A$ и $B$ у обеих границ расплава различные, в зоне расплава имеет место градиент концентрации. При достаточно длительной выдержке один из компонентов $(A)$ может полностью раствориться (рис. 26, в). Далее происходит растворение второго компонента до тех пор, пока концентрация всего расплава не приобретет величину $C_{B T}$ (рис. 22 ).

При общем понижении температуры (если вещество $A$ не растворено полностью (рис. $2 a)$ ) линия температур пересекает линию локальных ликвидусов как у границы $A \mid L$, так и у границы $L \mid B$ и создает в этих местах переохлаждение веществ $A$ и $B$ соответственно. Таким образом, происходит одновременный направленный рост двух автоэпитаксиальных слоев. Кристаллизация в сторону понижения локальных температур ликвидуса вызывает уменьшение наклонов линий локальных ликвидусов, и в конце процесса происходит кристаллизация состава, близкого к эвтектическому. Учитывая влияние кристаллических веществ $A$ и $B$ получим структуру

$$
A \underset{\rightarrow}{\rightarrow} \mid e\} e\left|B^{\prime}\right| B .
$$

Если вещество $A$ растворилось полностью, а содержание вещества $B$ у внешней границы расплава меньше эвтектического (рис. 2б), в структуре отсутствует слой исходного вещестBa $A$ :

$$
\underset{\rightarrow}{A^{\prime} \xi e \xi e\left|B^{\prime}\right| B .}
$$

Если у внешней границы расплава из-за диффузии содержание вещества $B$ станет больше эвтектического (рис. $28)$, не происходит первичной кристаллизации вещества $A$. Получим структуру

$$
\underset{\leftarrow}{e}\left|B^{\prime}\right| B .
$$

Остальные структуры, образующиеся в случае $T_{a}=T_{b}$, приведены в табл. 6 .

2) $T_{a}=T_{b}$. Изменения, происходящие в этом случае до начала понижения температуры, можно объяснить с помощью рис. 3. Вещество, находящееся при более низкой температуре, может растворяться только до тех пор, пока в момент $\tau_{3}=\tau_{e}$ фронт растворения не до-

Рис. 3. Изменения, происходящие в слое расплава при контактном сплавленин до поннжения температуры.

$a$ - распределение температуры; 6 - распределение концентраций в разные моменты времени; $\theta$ - распределение локальных температур ликвидуса и солидуса в разные моменты времени.

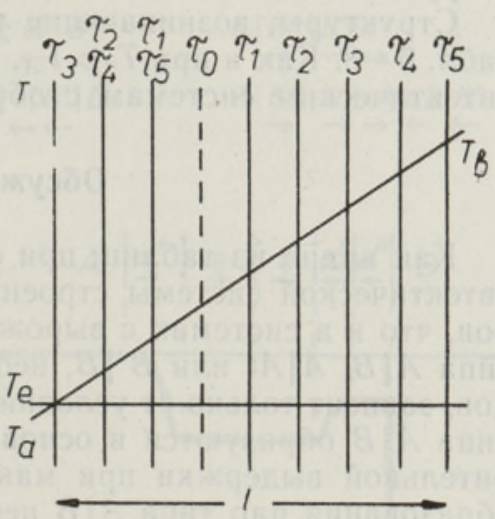

a
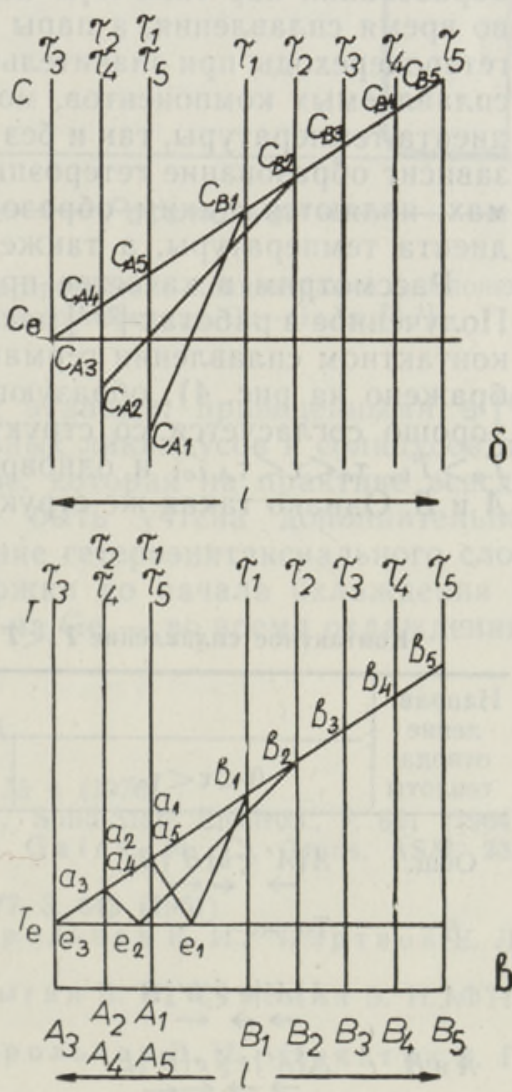
стигнет эвтектической температуры $T=T_{e}$ (рис. $3 a$ ). Соответственно понижению температуры на границе расплава и твердого вещества (например $A \mid L$ ) увеличивается равновесная концентрация $C_{A T}$, и если $T=T_{e}$, будет $C_{A T}=C_{e}$ (рис. $\left.3 б\right)$. С этого момента слой расплава ведет себя так же, как и слой растворителя в случае метода движущегося растворителя: из-за диффузии вещества $B$ в расплаве в сторону $A$ у границы $A \mid L$ создается пересыщение вещества $B$ и происходит направленная кристаллизация гетероэпитаксиального слоя $B^{D}$ на исходное вещество $A$. Таким образом, расплавленный слой может продвнгаться через весь слой исходного вещества $B$ в сторону более высоких температур, оставляя за собой перекристаллизованный слой $B^{D}$.

Структуры, возникающие при контактном сплавлении, приведены в табл. $6-8$. Как и при $T_{a}>T_{A}$, приведенные структуры относятся также к эвтектическим системам с образованием твердых растворов.

\section{Обсуждение результатов}

Как видно из таблиц, при образовании сплавляемыми компонентами эвтектической системы строение структуры зависит от тех же параметров, что и в системах с вырожденной эвтектикой [ $\left.{ }^{1}\right]$, но образование пар типа $A|B, A| A^{\prime}$ или $B^{\prime} \mid B$, необходимых для образования гетеропереходов, зависит только от условий сплавления, а не охлаждения. Пары слоев типа $A \mid B$ образуются в основном уже в ходе сплавления (т. е. предварительной выдержки при максимальной температуре процесса). Для образования пар типа $A \mid B$ необходимо наличие градиента температуры во время сплавления, а пары типа $A \mid A^{\prime}$ и $B^{\prime} \mid B$, позволяющие получать гетеропереходы при значительных областях твердых растворов на основе сплавляемых компонентов, могут образовываться как при наличии градиента температуры, так и без него. Основными параметрами, от которых зависит образование гетероэпитаксиальных слоев в эвтектических системах, являются, таким образом, наличие, направление и величина градиента температуры, а также длительность выдержки при сплавлении.

Рассмотрим в качестве примера сплавной гетеропереход $\mathrm{Ge}-\mathrm{GaAs}$. Полученное в работах $\left[{ }^{2-4}\right]$ распределение концентраций компонентов при контактном сплавлении германия и арсенида галлия (схематически изображено на рис. 4), образующих по данным [ $\left.{ }^{9}\right]$ эвтектическую систему, хорошо согласуется со структурой, приведенной в табл. 7 для случая $T_{a}>T_{b}, \tau_{e}<\tau<\tau_{A}$ tot и одновременного отвода теплоты в направлениях $A$ и $B$. Однако такая же структура может возникнуть и при общем пони-

Таблица 7

Контактное сплавление $\boldsymbol{T}_{e}<\boldsymbol{T}<\boldsymbol{T}_{A}, \boldsymbol{T}_{a}>\boldsymbol{T}_{b}$. Имеются твердые фазы $\boldsymbol{A}$ и $\boldsymbol{B}$

\begin{tabular}{|c|c|c|c|}
\hline \multirow{2}{*}{$\begin{array}{c}\text { Направ- } \\
\text { ление } \\
\text { отвода } \\
\text { теплоты }\end{array}$} & \multicolumn{3}{|c|}{ Слоистые структуры } \\
\hline & $0<\tau<\tau_{e}$ & $\tau_{e}<\tau<\tau_{A} t \circ t$ & $\tau_{A} t o t<\tau<\infty$ \\
\hline Общ. & $\stackrel{A \mid A^{\prime}}{\rightarrow} \underset{\leftarrow}{\leftarrow} \stackrel{B^{\prime} \mid B}{\leftarrow}$ & $\begin{array}{c}A\left|A^{\prime} \zeta e\right| A^{\prime}\left|A^{D}\right| B \\
\rightarrow \leftarrow \leftarrow \leftarrow\end{array}$ & $\begin{array}{l}e\left|A^{\prime}\right| A^{D} \mid B \\
\leftarrow \leftarrow \leftarrow\end{array}$ \\
\hline$B$ & То же & То же & То же \\
\hline$A$ & $\begin{array}{l}\left.A\left|A^{\prime}\right| e\right\} B^{\prime} \mid B \\
\rightarrow \rightarrow\end{array}$ & $\begin{array}{c}A\left|A^{\prime}\right| e \zeta A^{\prime}\left|A^{D}\right| B \\
\rightarrow \leftrightarrow\end{array}$ & $\begin{array}{l}\left.A^{\prime} 3 e\right\} A^{\prime}\left|A^{D}\right| B \\
\rightarrow \stackrel{\leftarrow}{\rightarrow} \leftarrow\end{array}$ \\
\hline$A$ и $B$ & $\begin{array}{c}A\left|A^{\prime}\right| e \leq e\left|B^{\prime}\right| B \\
\rightarrow \leftrightarrow \leftarrow\end{array}$ & $\begin{array}{c}\left.A\left|A^{\prime}\right| e\right\} e\left|A^{\prime}\right| A^{D} \mid B \\
\rightarrow \rightarrow \leftarrow \leftarrow\end{array}$ & 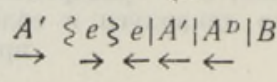 \\
\hline
\end{tabular}


Контактное сплавление $\boldsymbol{T}_{e}<\boldsymbol{T}<\boldsymbol{T}_{A}, \boldsymbol{T}_{a}<\boldsymbol{T}_{b}$. Имеются твердые фазы $\boldsymbol{A}$ и $\boldsymbol{B}$

\begin{tabular}{|c|c|c|c|}
\hline \multirow{2}{*}{$\begin{array}{c}\text { Направ-я } \\
\text { ление } \\
\text { отвода } \\
\text { теплоты }\end{array}$} & \multicolumn{3}{|c|}{ Слонстые структуры } \\
\hline & $0<\tau<\tau_{e}$ & $\tau_{e}<\tau<\tau_{B} t o t$ & $\tau_{B} t_{o t}<\tau<\infty$ \\
\hline Общ. & $\begin{array}{c}A\left|A^{\prime}\right| e \xi B^{\prime} \mid B \\
\rightarrow \rightarrow\end{array}$ & $\begin{array}{c}\left.A\left|B^{D}\right| B^{\prime} \mid e\right\} B^{\prime} \mid B \\
\quad \rightarrow \rightarrow \rightarrow\end{array}$ & $\stackrel{A\left|B^{D}\right| B^{\prime} \mid e}{\rightarrow} \rightarrow \underset{ }{\rightarrow}$ \\
\hline$B$ & $\begin{array}{c}\left.A \mid A^{\prime}\right\} e\left|B^{\prime}\right| B \\
\rightarrow \leftarrow \leftarrow\end{array}$ & 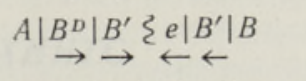 & $\begin{array}{c}A\left|B^{D}\right| B^{\prime}\left\{e\left\{B^{\prime}\right.\right. \\
\rightarrow \rightarrow \leftarrow\end{array}$ \\
\hline A & $\begin{array}{c}A\left|A^{\prime}\right| e \xi B^{\prime} \mid B \\
\rightarrow \rightarrow \leftarrow\end{array}$ & $\stackrel{A\left|B^{D}\right| B^{\prime}\left|e \xi B^{\prime}\right| B}{\rightarrow} \rightarrow \underset{\leftarrow}{\rightarrow} \stackrel{\leftarrow}{\leftarrow}$ & $\begin{array}{c}A\left|B^{D}\right| B^{\prime} \mid e \\
\rightarrow \rightarrow\end{array}$ \\
\hline$A$ и $B$ & $\begin{array}{c}A\left|A^{\prime}\right| e\left\{e\left|B^{\prime}\right| B\right. \\
\quad \rightarrow \rightarrow \leftarrow \leftarrow\end{array}$ & $\begin{array}{c}A\left|B^{D}\right| B^{\prime} \mid e\left\{e\left|B^{\prime}\right| B\right. \\
\rightarrow \rightarrow \leftarrow \leftarrow\end{array}$ & $\begin{array}{c}\left.\left.A\left|B^{D}\right| B^{\prime} \mid e\right\} e\right\} B^{\prime} \\
\rightarrow \rightarrow \rightarrow \leftarrow\end{array}$ \\
\hline
\end{tabular}

жении температуры из-за выделения скрытой теплоты на фронтах кристаллизации. Выделение скрытой теплоты кристаллизации, по-видимому, может создать некоторый максимум температуры в области расплавленной зоны, и в этом случае структуры, приведенные в таблицах для общего понижения температуры, нужно заменить структурами, соответствующими отводу теплоты в направлениях $A$ и $B$. Этапы формирования гетероперехода, полученные с помощью метода локальных ликвидусов и солидусов, в общих чертах совпадают с этапами, приведенными в [4]. Различием является то, что метод локальных ликвидусов и солидусов не учитывает диффузию в твердом веществе, которая на практике всегда имеет место и, следовательно, должна быть учтена дополнительно. Согласно нашим рассуждениям, нарастание гетероэпитаксиального слоя $\mathrm{Ge}$ на GaAs начинается во время выдержки до начала охлаждения и нарастание автоэпитаксиального слоя Ge на Ge - во время охлаждения.

\section{Л И ТЕ РА Т У Р А}

1. Коппел Х., Изв. АН ЭССР. Хим. Геол., 25, № 4 (1976).

2. Rediker, R. H., St opek, S., W a rd, J., Solid-State Electron., 7, 621 (1964).

3. Mroczkowski, R. S., Lavine, M. C., Gatos, H. C., Trans. ASM, 233, 456 (1965).

4. Ч и ст яков Ю. Д., М а т сон Э. А., ДАН, 177, 3, 643 (1967).

5. Альферов Ж. И., Андреев В. М., Корольков В. И., Портной Е. Л., Я к о венко А. А., ФТП, 3, 930 (1969).

6. Альферов Ж. И., Андреев В. М., Мурыгин В. И., Стремин В. И., ФТП, 3,1470 (1969).

7. Альферов Ж. И., Андреев В. М., Корольков В. И., Никитин В.Г., Я к о в н ко А. А., ФТП, 4,578 (1970). 
8. Ч и стяков Ю. Д., Сб. науч. тр. по проблемам микроэлектроники. Эпитаксия, 4, 36 (1969).

9. Гл аз ов В. М., М ал ют ин а Г. Л., ЖНХ, 8, 8, 1921 (1963).

Ннститут физики

Академии наук Эстонской ССР
Поступила в редакцию 4/III 1974

H. KOPPEL

\section{SULANDATUD HETEROSIIRETE STRUKTUURIST EUTEKTILISTE SUSTEEMIDE KORRAL}

Kvalitatiivselt on vaadeldud kihiliste struktuuride ehituse sõltuvust temperatuurigradiendi olemasolust, suunast ja suurusest eelneval kuumutamisel enne jahutamise algust, eelneva kuumutamise kestusest, soojuse äravoolu suunast ja temperatuurigradiendi suurusest jahutamise ajal ning jahutamise kiirusest, kui komponendid moodustavad eutektilise süsteemi. On esitatud mitmesugustel tingimustel moodustuvaid kihilisi struktuure.

\section{H. KOPPEL}

\section{ON THE STRUCTURE OF ALLOYED HETEROJUNCTIONS IN THE CASE OF EUTECTIC SYSTEMS}

The dependences of the junction's layer structure on the value and direction of temperature gradient during alloying and cooling, the duration of alloying and the cooling rate, are considered. The layer structures formed at various conditions in the case of eutectic systems are presented. 